\title{
Determination of the unsaturated disaccharides of hyaluronic acid in equine synovial fluid by high-performance liquid chromatography and fluorescence detection
}

\author{
Kaisa Aaltonen", Tytti Niemelä, Satu Sankari and Riitta-Mari Tulamo
}

\begin{abstract}
Background: The purpose of this study was to develop and validate an analytical method to determine the presence of hyaluronic acid derived disaccharides in equine synovial fluid.

Findings: A high-performance liquid chromatography method for the determination of hyaluronic acid derived unsaturated disaccharides in equine synovial fluid was developed and validated. The method is based on the measurement of unsaturated disaccharides released by digestion of linear hyaluronic acid molecules. The method showed linearity $\left(r^{2}=0.996\right)$ over the full working concentration range $0.89-30 \mathrm{mg} / \mathrm{l}$. Relative standard deviation of intra- and inter-day precision ranged from of $4.3-6.7 \%$ and $7.1-7.8 \%$ respectively. The detection limit was $0.3 \mathrm{mg} / \mathrm{l}$ corresponding to $20 \mathrm{mg} / \mathrm{l}$ in synovial fluid. Accuracy of the assay was $97-103 \%$. This method was evaluated by determining the concentration of unsaturated disaccharides from hyaluronic acid in synovial fluid of horses with lameness in the metacarpo-/metatarsophalangeal joint localized with positive response to intra-articular anesthesia.

Conclusions: The described method is valid for determination of hyaluronic acid derived disaccharides in equine synovial fluid. This method was applied to a larger research project dealing with a new form of intra-articular therapy in horses with arthritic diseases.
\end{abstract}

Keywords: Equine, High-performance liquid chromatography, Hyaluronic acid, Synovial fluid

\section{Findings}

Hyaluronic acid (HA) is a linear polysaccharide formed of disaccharide units which are linked together to form the HA chain. The molecular mass of HA can be as high as $10^{7}$ Daltons depending on the number of repeating disaccharide units in the chain [1].

The viscoelasticity of synovial fluid (SF) is attributable to its rich HA content and this molecule serves as the principal lubricant of synovial soft tissues [2]. In arthritis, the synthesis of HA is decreased and degradation enhanced. This results in the reduction of HA concentration and molecular mass, and diminished viscoelasticity in SF [3]. The concentration of HA can be used as a diagnostic marker for arthritis where a significantly

\footnotetext{
* Correspondence: kaisa.aaltonen@helsinki.fi

Department of Equine and Small Animal Medicine, University of Helsinki, P.O. Box 57, 00014 Helsinki, Finland
}

lower level of HA compared to that of control horses has been reported [4]. The effects of various intraarticular treatments could also be monitored using the concentration of HA in the SF.

Various methods have been described for the estimation of the quantity of HA in SF by using either polymeric or fragmented molecules, such as: size-exclusion chromatography, fluorophore-assisted carbohydrate electrophoresis, enzyme-linked immunosorbent, radiometric binding, and colorimetric assays [5-9]. Analysis of high-molecular mass $\mathrm{HA}$ with different degree of polymerization is challenging. The unavailability of size exclusion columns with high exclusion limits causes difficulties in the analysis of very long HA chains. By digestion of HA to unsaturated disaccharides and labelling with a hydrophobic fluorescent agent, the analysis is enabled by reverse-phase high-performance liquid 
chromatography (HPLC) which is reported to offer good resolution $[10,11]$. Labeling by a fluorescent molecule also increases detection sensitivity and specificity significantly [12]. Reverse-phase HPLC has been used to analyze HA-disaccharides in biological samples [10], however to our knowledge the method is not validated for equine SF.

The aim of this study was to develop and validate an accurate and specific HPLC method, with low sample volume, for determination of the concentration of unsaturated disaccharides of $\mathrm{HA}(\triangle \mathrm{Di}-\mathrm{HA})$ in equine SF and apply the method as part of a larger study. The indirect method to determine the total amount of HA is based on the analysis of $\triangle \mathrm{Di}-\mathrm{HA}$ produced by digestion. To evaluate the suitability of the method, $\triangle \mathrm{Di}-\mathrm{HA}$ was quantified in SF of the equine metacarpo-/metatarsophalangeal (MCP/MTP) joints.

Twenty eight equine SF samples were analyzed. Samples were obtained by performing routine aseptic arthrocentesis with an 18 gauge $/ 3.8 \mathrm{~cm}$ needle which was introduced into the MCP/MTP joint through the lateral sesamoidean ligament. SF sample was withdrawn and $10 \mathrm{ml}$ anesthetic solution, mepivacaine hydrochloride (Scandicain ${ }^{\circ}$ Astra Zeneca, Zug, Switzerland) was injected intra-articularly (IA). The horses were free of lameness when evaluated $10 \mathrm{~min}$ after IA-anesthesia which verified that lameness was originating from this joint. The joints were medicated and results are reported elsewhere.

SF samples were centrifuged for $10 \mathrm{~min}$ at $4000 \mathrm{x} g$ at $4^{\circ} \mathrm{C}$. Supernatants were removed and frozen at $-80^{\circ} \mathrm{C}$ until analyzed. All samples were analyzed in duplicate.

Before analysis, SF sample was thawed and diluted 1:1 in water. An aliquot $(5 \mu \mathrm{l})$ was digested with chondroitinase $\mathrm{ABC}$ (cABC) from Proteus vulgaris (Sigma Aldrich, St Louis, MO, USA) (25 mU) in $50 \mathrm{mM}$ ammonium acetate buffer, $\mathrm{pH} 6.8$ at $37^{\circ} \mathrm{C}$ for $20 \mathrm{~h}$. After digestion, $900 \mathrm{ng}$ internal standard $\triangle$ Di-UA-2S (Sigma Aldrich, St Louis, MO, USA) was added and the mixture was filtrated using a centrifugal filter (Ultrafree-MC centrifugal filter device, Amicon Bioseparations, Millipore, MA, USA) at $12000 \times g$ for $45 \mathrm{~min}$ at $4^{\circ} \mathrm{C}$. The filtrate was evaporated to dryness in a vacuum evaporator.

Dried disaccharides were derivatized as described previously [13,14]. Briefly, $5 \mu \mathrm{l}$ of $0.1 \mathrm{M} 2$-aminoacridone (AMAC) was added in glacial acetic acid / dimethyl sulfoxide (DMSO) (3:17, v/v) and incubated at room temperature for $15 \mathrm{~min}$. Then $5 \mu$ freshly prepared $1 \mathrm{M}$ cyanoborohydride was added and the mixture was incubated at $45^{\circ} \mathrm{C}$ for $4 \mathrm{~h}$. Finally, $190 \mu \mathrm{l}$ of $50 \%$ (v/v) DMSO was added and $10 \mu \mathrm{l}$ of clear filtrate was analyzed.

For standards, $1 \mathrm{mg} \triangle \mathrm{Di}-\mathrm{HA}$ (Sigma Aldrich, St Louis, MO, USA) and $\triangle$ Di-UA-2S were dissolved in $1 \mathrm{ml} 90 \%$ methanol separately. Working solutions were made by diluting the stock solutions 1:10 in 90\% methanol. Standards were stored at $-20^{\circ} \mathrm{C}$. $\triangle \mathrm{Di}-\mathrm{HA}$ was taken to yield final concentrations between $0-30 \mathrm{mg} / \mathrm{l}$ and $\Delta$ Di-UA-2S $4.5 \mathrm{mg} / \mathrm{l}$. The standards were evaporated and derivatized like samples and they were made weekly.

Chromatography was performed on an 1100 series HPLC (Agilent Technologies Co., Waldbronn, Germany) and a fluorescence detector (excitation at $442 \mathrm{~nm}$ and emission $520 \mathrm{~nm}$ ). The column used was a Zorbax Eclipse XDB-C18 $(4.6 \mathrm{~mm} \times 250 \mathrm{~mm}, 5 \mu \mathrm{m})$ (Agilent Technologies Co., Little Falls (Wilmington), Delaware, USA) fitted with the guard column of the same type. The flow rate was $1 \mathrm{ml} / \mathrm{min}$. The column was first equilibrated with $98 \%$ buffer $(100 \mathrm{mM}$ ammonium acetate, $\mathrm{pH}$ 5.6) (A) and $2 \%$ methanol (B) for $10 \mathrm{~min}$. A linear gradient was run starting with the initial condition for 2 min to $60 \%$ of eluent B in $40 \mathrm{~min}$. Finally the column was flushed with $60 \%$ eluent B for $9 \mathrm{~min}$. Column temperature was held at $22^{\circ} \mathrm{C}$. Total run time was $61 \mathrm{~min}$.

A calibration curve was obtained by plotting peak areas of ten $\triangle \mathrm{Di}-\mathrm{HA}$ standards against their concentrations followed by linear regression analysis calculated using the method of least squares. The peaks were identified by comparing their retention times with those obtained from standards and confirmed by spiking technique. The samples were quantified using the peak area of $\triangle \mathrm{Di}-\mathrm{HA}$ and corrected using the ratio of a peak area of the internal standard in the sample and a peak area of the internal standard in the standard solutions.

Chondroitin sulfate (CS) may be present in small amounts in SF [15]. Chondroitinase ABC degrades HA as well as CS to the unsaturated disaccharides. Nonsulfated CS-disaccharides ( $\triangle \mathrm{Di}-0 \mathrm{~S})$ may interfere with the analysis of HA-disaccharides by eluting with the same retention time by HPLC whereas sulfated CSdisaccharides separate from the peak of the $\triangle \mathrm{Di}-\mathrm{HA}$ because of decreasing hydrophobicity. The current assay was compared for resolution of CS-disaccharides by digesting chondroitin sulfate sodium salt from shark cartilage (Sigma-Aldrich, St. Louis, MO, USA) by cABC using the same conditions as for SF samples and analyzed with the same method.

The intra-day precision was established by analyzing two equine SF samples five times in one day under the same conditions. The inter-day precision was determined by analyzing these same samples over five different days. The precision was expressed as relative standard deviation (RSD\%).

The analytical sensitivity of the method was expressed as the limit of quantification defined as the minimum amount of $\triangle \mathrm{Di}-\mathrm{HA}$ that resulted in a peak height that was ten times that of baseline noise and the limit of detection, which has a peak height three times that of baseline noise. 


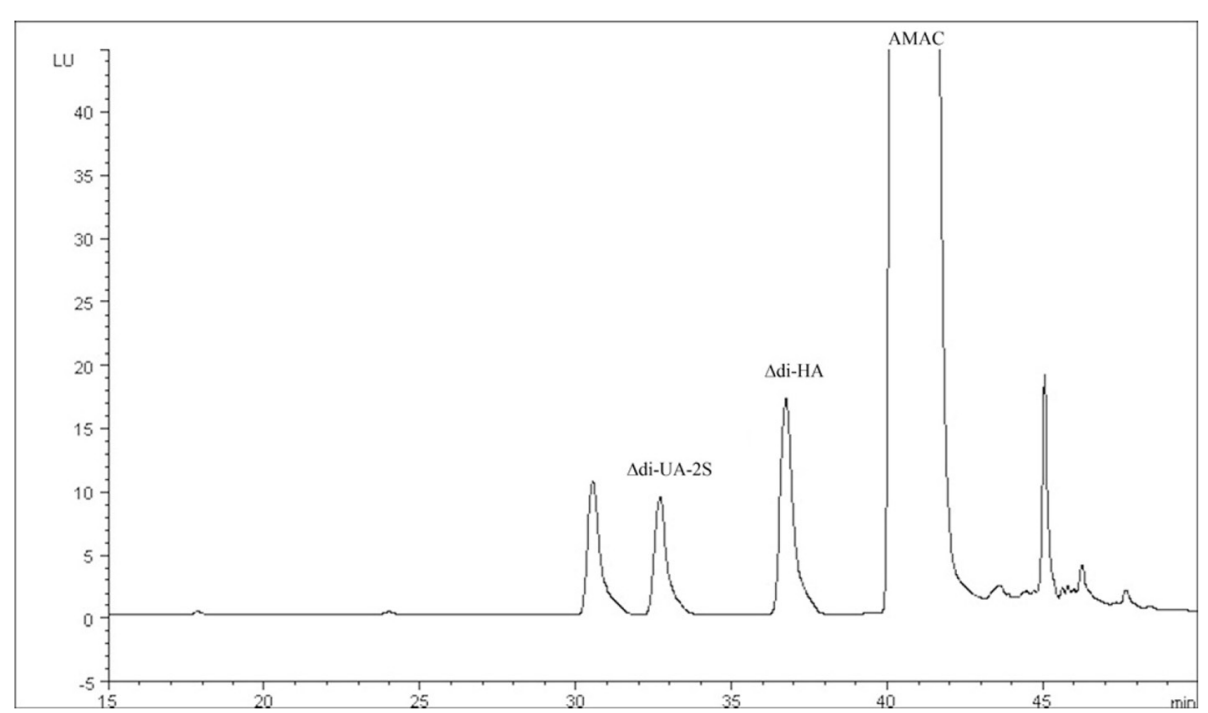

Figure 1 Chromatogram of AMAC-labelled $\triangle \mathrm{Di}$-HA, and internal standard, $\triangle \mathrm{Di}$-UA-2S. Chromatography performed on a reverse-phase column with a linear gradient of $2-60 \%$ acetonitrile. Disaccharides were detected by fluorescence excitation at $442 \mathrm{~nm}$ and emission at $520 \mathrm{~nm}$.

The accuracy of the method was assessed by spiking two different concentrations of $\triangle \mathrm{Di}-\mathrm{HA}$ in the known equine SF and comparing measured and actual values. The percentage recovery was reported as accuracy.

A typical chromatogram is shown in Figure 1. Peaks of $\triangle$ Di-UA-2S and $\triangle$ Di-HA separated with good resolution. The retention times were $32.1 \mathrm{~min}$ and $36.3 \mathrm{~min}$ respectively. The CS-disaccharides, fragmented from shark cartilage with $\mathrm{CABC}$, eluted with different retention times. The amount of total CS in equine SF is around $30 \mathrm{mg} / \mathrm{l}$ in normal rested and exercised horses and the portion of $\triangle \mathrm{Di}-0 \mathrm{~S}$ of total CS is shown to be low [15]. In this method, the concentration of $\triangle \mathrm{Di}-0 \mathrm{~S}$ was not studied because its interference to the concentration of HA-disaccharides is negligible.

The calibration curve for $\triangle \mathrm{Di}-\mathrm{HA}$ standards showed a linear response over the full concentration range (0.89$30 \mathrm{mg} / \mathrm{l})$ with a correlation coefficient $r^{2}=0.996$. The intra-day and inter-day precisions (RSD\%) were 4.3$6.7 \%$ and $7.1-7.8 \%$ respectively. The detection limit was $0.3 \mathrm{mg} / \mathrm{l}$ corresponding to $20 \mathrm{mg} / \mathrm{l}$ in SF. The limit of quantitation was $0.89 \mathrm{mg} / \mathrm{l}$ corresponding to $70 \mathrm{mg} / \mathrm{l}$ in SF. The accuracy of the assay was $97-103 \%$.

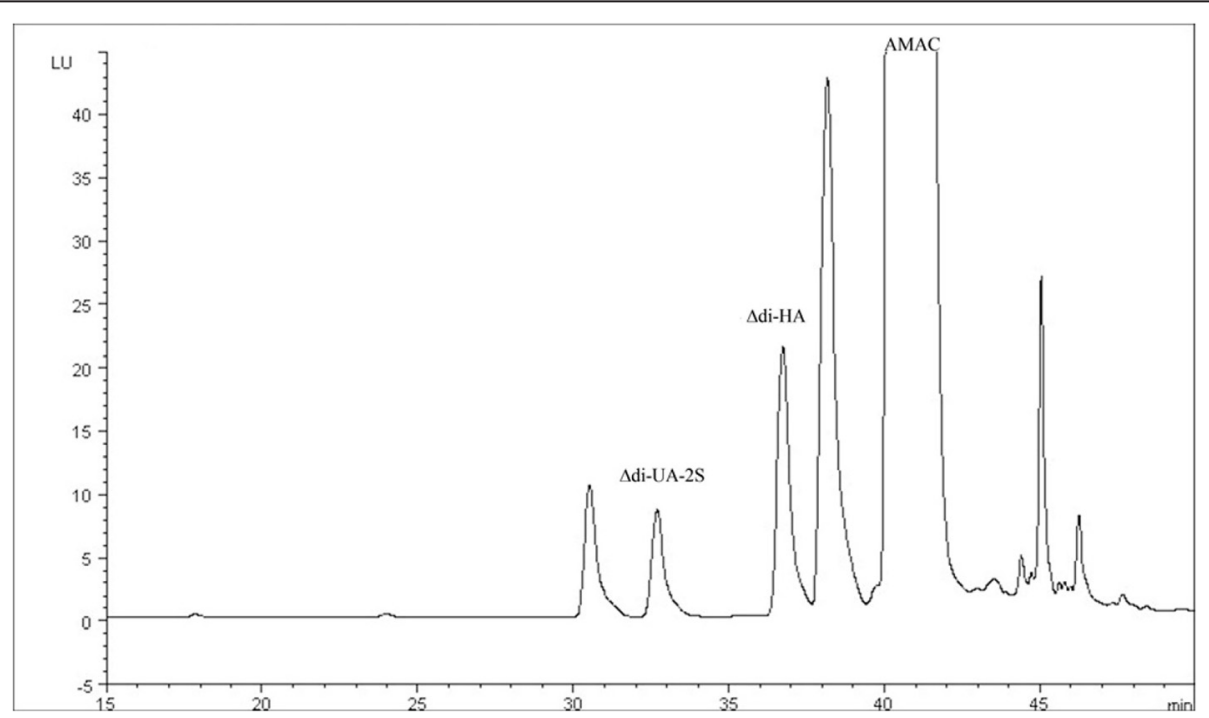

Figure 2 Typical chromatogram of AMAC-labelled $\triangle \mathrm{Di}$-HA fragmented by $C A B C$ from hyaluronic acid in SF of the equine metacarpo-/metatarsophalangeal joint and internal standard, $\triangle \mathrm{Di}-\mathrm{UA}-2 \mathrm{~S}$. 
Figure 2 shows a typical chromatogram of unsaturated disaccharides produced from $\mathrm{HA}$ in equine SF. The mean $\triangle \mathrm{Di}-\mathrm{HA}$ concentration $(n=28)$ was $832 \mathrm{mg} / \mathrm{l}$ (range 364-1980 mg/l) in SF from the MCP/MTP joint in horses with lameness.

$\triangle \mathrm{Di}$-HA has been successfully analyzed by reversephase HPLC with pre- $[10,11]$ and postcolumn [16] derivatization. Among others, danzylhydrazine [12], 2aminopyridine [17], and AMAC [14] have been used as pre-column reagents. The reaction of AMAC with the $\triangle \mathrm{Di}-\mathrm{HA}$ is simple and rapid. Hydrophobic AMAClabeling permits separation by reverse-phase HPLC eliminating the need for ion-pair reagents [18]. AMACderivatives have been shown to be stable for one month at room temperature, for longer periods of time, storage at $-80^{\circ} \mathrm{C}$ is recommended [19].

In summary, the present method for quantifying $\triangle \mathrm{Di}-\mathrm{HA}$ in equine SF was validated for linearity, accuracy, inter-day, and intra-day precision, specificity, detection and quantitation limits. Reverse-phase HPLC used here provides a useful, accurate, and specific method for measurement of the AMAC-labelled unsaturated disaccharides. Because of high content of HA in SF, just a few microliters of SF are required for the present method. Since only a conventional HPLC apparatus with a fluorescence detector is required, this method can be employed in most laboratories. This analytical method was successfully used to monitor disaccharides derived from $\mathrm{HA}$ in equine SF.

\begin{abstract}
Abbreviations
AMAC: 2- aminoacridone; $C A B C$ : chondroitinase ABC; CS: chondroitin sulfate; DMSO: dimethyl sulfoxide; HA: hyaluronic acid; HPLC: high-performance liquid chromatography; IA: intra-articularly; MCP/MTP: metacarpo-/ metatarsophalangeal joint; RSD: relative standard deviation; SF: synovial fluid; $\triangle$ Di-OS: nonsulfated unsaturated disaccharides of chondroitin sulfate, 2-acetamido-2-deoxy-3-O-( $\beta$-D-gluco-4-enepyranosyluronic acid)D-galactose; $\triangle \mathrm{Di}-\mathrm{HA}$ : unsaturated disaccharides of hyaluronic acid, 2-acetamido2-deoxy-3-O-( $\beta$-D-gluco-4-enepyranolyluronic acid)-D-glucose; $\triangle \mathrm{Di}$-UA 2S: unsaturated chondroitin disaccharide, 2-acetamido-2-deoxy-3-O-(2-O-sulfo- $\beta$ D-gluco-4-enepyranosyluronic acid)-D-galactose.
\end{abstract}

\section{Competing interests}

The authors declare that they have no competing interests.

\section{Authors' contributions}

KA carried out the chromatographic studies and drafted the manuscript. TN collected the samples and participated in writing of the manuscript. SS supported writing and design of the study. R-MT participated in the design of the study and helped to draft the manuscript. All authors have read and approved the final version of the manuscript.

Received: 8 October 2014 Accepted: 22 January 2015

Published online: 04 March 2015

\section{References}

1. Fraser JRE, Laurent TC, Laurent UBG. Hyaluronan: its nature, distribution, functions and turnover. J Int Med. 1997;242:27-33.

2. Balatz $E$, Denlinger J. Viscosupplemention: a new concept in the treatment of osteoarthritis. J Rheumatol Suppl. 1993;39:3-9.
3. Goldenberg VM, Buckwalter JA. Hyaluronans in the treatment of osteoarthritis of the knee: evidence for disease-modifying activity. Osteoarthritis Cartilage. 2005;13:216-24.

4. Tulamo R, Houttu J, Tupamäki A, Salonen M. Hyaluronate and large molacular weight proteoglycans in synovial fluid from horses with various arthritides. Am J Vet Res. 1996;57:932-7.

5. Saari H, Konttinen YT, Tulamo R-M, Antti-Poika I, Honkanen V. Concentration and degree of polymerization of hyaluronate in equine synovial fluid. Am J Vet Res. 1989;50:2060-3.

6. Canapp SO, Brown MP, Lewis DD, Hernandez J, Merritt KA, Tran-Son-Tay R. Examination of synovial fluid and serum following intravenous injections of hyaluronan for the treatment of osteoarthritis in dogs. Vet Comp Orthop Traumatol. 2005:18:169-74

7. Plickert HD, Bondzio A, Einspanier R, Brunnberg L. Hyaluronic acid concentrations in synovial fluid of dogs with different stages of osteoarthritis. Res Vet Sci. 2013;94:728-34.

8. Popot M, Bonnaire Y, Guechot J, Toutain P. Hyaluronan in horses: physiological production rate, plasma and synovial fluid concentrations in control conditions and following sodium hyaluronate administration. Equine Vet J. 2004;36:482-7

9. Ishimaru Jl, Ogi N, Mizuno S, Goss A. Quantitation of chondroitin-sulfates, disaccharides and hyaluronan in normal, early and advanced osteoarthritic sheep temporomandibular joints. Osteoarthritis Cartilage. 2001;9:365-70.

10. Ambrosius M, Kleesiek K, Götting C. Quantitative determination of the glucosaminoglycan delta-disaccharide composition of serum, platelets and granulocytes by reversed-phase high-performance liquid chromatography. J Chromatogr A. 2008;1201:54-60.

11. Volpi N. High-performance liquid chromatography and on-line mass spectrometry detection for the analysis of chondroitin sulfates/hyaluronan disaccharides derivatized with 2-aminoacridone. Anal Biochem. 2010;397:12-23

12. Volpi N. Hyaluronic acid and chondroitin sulfate unsaturated disaccharides analysis by high-performance liquid chromatography and fluorometric detection with dansylhydrazine. Anal Biochem. 2000;277:19-24.

13. Jackson P. Polyacrylamide gel electrophoresis of reducing saccharides labeled with the fluorophore 2-aminoacridone: Subpicomolar detection using an imaging system based on a cooled charge-coupled device. Anal Biochem. 1991;196:238-44.

14. Kitagawa H, Kinoshita A, Sugahara K. Microanalysis of glycosaminoglycanderived disaccharides labeled with the fluorophore 2-aminoacridone by capillary electrophoresis and high-performance liquid chromatography. Anal Biochem. 1995;232:114-21.

15. Brown M, Trumble T, Plaas A, Sandy J, Romano B, Hernandez D, et al. Exercise and injury increase chondroitin sulfate chain length an decrease hyaluronan chain length in synovial fluid. Osteoarthris Cartilage. 2007;15:1318-25

16. Shibata T, Murakami K-I, Kubota E, Maeda H. Glycosaminoglycan components in temporomandibular joint synovial fluid as markers of joint pathology. J Oral Maxillofac Surg. 1998:56:209-13.

17. Narita H, Takeda Y, Takagaki $K$, Nakamura T. Identification of glycosaminoglygans using high-pergformance liquid chromatography on a hydroxyapatie column. Anal Biochem. 1995;232:133-6.

18. Yang B, Chang Y, Weyers AM, Sterner E, Linhardt RJ. Disaccharide analysis of glycosminoglygan mixtures by ultra-high-performance liquid chromatography-mass spectrometry. J Chromatogr A. 2012;1225:91-8.

19. Militsopoulou M, Lamari FN, Hjerpe A, Karamanos NK. Determination of twelve heparin- and heparan sulfate-derived disaccharides as 2aminoacridone derivatives by capillary zone electrophoresis using ultraviolet and laser-induced fluorescence detection. Electrophoresis. 2002;23:1104-9. 